\title{
Very Fast Schoenflies Motion Generator
}

\author{
Vincent NABAT and María de la $\mathrm{O}$ \\ RODRIGUEZ \\ Fundación FATRONIK \\ Pol. Ibaitarte 1, Apdo. de Correos 160 \\ 20870 Elgoibar (Guipúzcoa), Spain
}

nabat@lirmm.fr,mrodriguez@fatronik.com

\author{
Olivier COMPANY and François \\ PIERROT \\ LIRMM \\ CNRS - University of Montpellier II \\ 161 rue Ada, 34392 Montpellier Cedex 5, \\ France \\ \{company, krut, pierrot\}@lirmm.fr
}

\author{
Pierre DAUCHEZ \\ LESIA \\ INSA Toulouse - EA 1687 \\ 135 avenue de Rangueil, 31077 \\ Toulouse Cedex 4, France \\ pierre.dauchez@insa-toulouse.fr
}

\begin{abstract}
This paper introduces a four-degree-of-freedom parallel manipulator producing Schonflies motions (three translations and one rotation). It has been developed with the goal of reaching very high speed. This paper shows that its architecture is particularly well adapted to high dynamics. Indeed, it is an evolution of Delta, $\mathrm{H} 4$ and $\mathrm{I} 4$ robots architectures: it keeps the advantages of these existing robots, while overcoming their drawbacks. In addition, this paper shows the dynamic modelling of the architecture that will be useful for a future dynamic control. Finally, experimental results are shown, and prove that the robot is able to reach high accelerations (13 G) and obtain a cycle time of $0.28 \mathrm{~s}$.
\end{abstract}

Index Terms - Schoenflies Motion, PKM, pick-and-place, Articulated traveling plate

\section{INTRODUCTION}

The first parallel mechanism is attributed to Gough with its well-known platform [1] and Stewart [2] created the famous flight simulator few years later. Thanks to actuators located close to the frame, the dynamics of these mechanisms is high compared to serial robots. These machines have six degrees of freedom $(d o f)$ and the range of their angular motion is limited.

However, all robotized tasks do not need six dof. Brogardh proposed a classification [3] giving the necessary number of $d o f$ for different industrial tasks. Generally, pickand-place needs four dof: three translations and one rotation around a vertical axis. These motions are named Schoenflies motions [4] or SCARA motions. The main industrial applications for these mechanisms are packaging, including picking, packing and palletizing tasks.

Delta robot [5], developed by Clavel at EPFL at the end of $80 \mathrm{~s}$, generates Schonflies motion and is well adapted to pick-and-place tasks because of its high dynamics. Indeed, actuators of this robot are fixed on the frame which minimizes moving parts masses. However, the rotational motion of this robot is obtained using a central RUPUR chain (R: Revolute, U: Universal, P: Prismatic, bold representing the actuated joint) which could suffer from a lack of stiffness at workspace extremities. In addition, this telescopic arm has a short service life.

Other lower mobility parallel mechanisms able to realize SCARA motions have been developed. For example, Angeles [6] proposed a four-dof parallel mechanism. In addition, EPFL developed Kanuk and Manta robots [7], and the machine tool
HITA STT which has been derived to pick-and place manipulator [8]. At last, authors proposed H4 [9], I4L [10] and I4R [11] introducing the articulated traveling plate concept (see Fig. 1).

This paper presents a new parallel manipulator based on H4 and I4 architectures: Par4. This mechanism has been developed with the aim of reaching very high speed and acceleration. Indeed, the second part of the paper presents the improvements of Par4 compared to I4 and H4. The third part exposes the dynamic moddling of this new architecture. The last part presents experimental results showing that the prototype is able to reach accelerations up to $13 \mathrm{G}$.

\section{DESCRIPTION OF PAR4}

The particularity of Par4 compared to H4 and I4 is its articulated traveling plate. It is composed of four parts: two main parts $(1,2)$ linked by two rods $(3,4)$ thanks to revolute joints (see Fig. 2). The shape of this assembly is a planar parallelogram and the internal mobility of the traveling plate is a PI joint [12] (circular translation) which produces the rotational motion about the vertical axis of the global robot. The range of this rotation is $\pm \pi / 4$. That's why, an amplification system has to be added in order to obtain a complete turn: $\pm \pi$. The amplification system can be made of gears or belt/pulleys. The chosen mechanism for the prototype is belt/pulleys with an amplification ratio $\rho=4$ (see Fig. 2b).

The overall architecture is similar to $\mathrm{H} 4$ or $\mathrm{I} 4 \mathrm{R}$ as described in [9] and [11]. Arms and forearms, made of carbon fiber, are taken from ABB Flexpicker robot. Par4 is a Deltalike mechanism. The key difference with the Delta robot is the use of four kinematic chains instead of three. In addition, it uses the concept of articulated traveling plate in order to avoid the central telescopic leg. The key difference with $\mathrm{H} 4$ and I4 robots will be explained in the following.

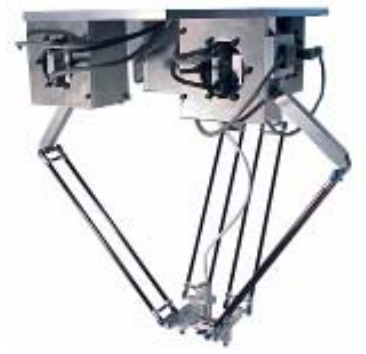

(a) $\mathrm{H} 4$ robot

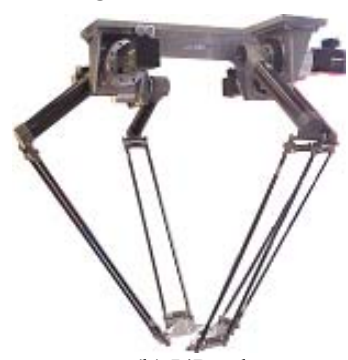

(b) I4R robot 


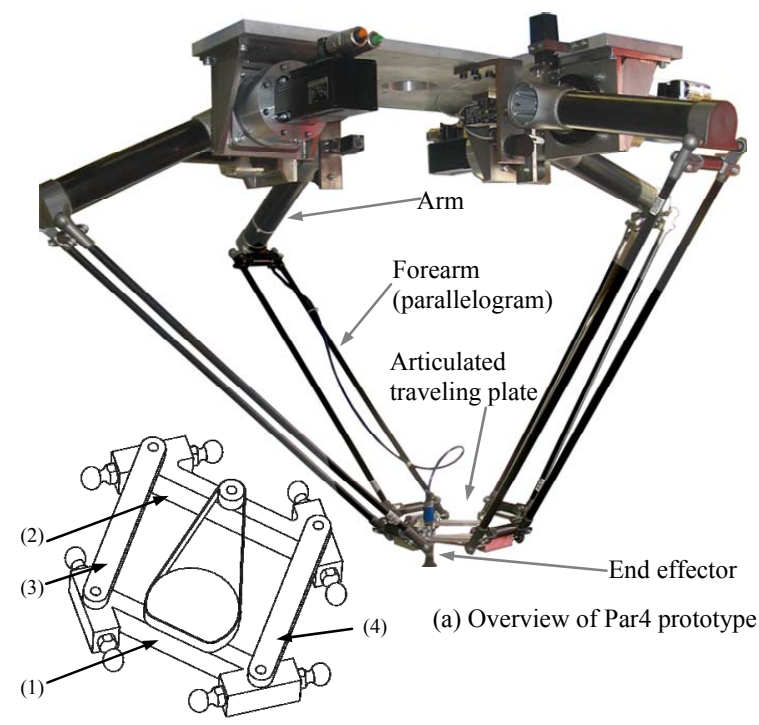

(b) Articulated traveling plate

Fig. 2 Par4 prototype

Remark about models for kinematics solutions:

As mentioned previously, the articulated traveling plate of Par4 produces a rotational translation. From a modeling point of view, it can be assimilated to H4. Thus, models for kinematics solutions (position and velocity) can be found in [9] and will not be presented here.

\section{WHY PAR4 INSTEAD OF H4 AND I4}

This new prototype has been developed in order to reach very high speed and acceleration, and to obtain a homogeneous behavior and a good stiffness in the whole workspace and for every direction. All these constraints can not be respected at the same time neither by $\mathrm{H} 4$ nor I4 for different reasons that are now explained.

\section{A. From 14 to Par4}

An advantage of this architecture is the good arrangement of actuators unlike H4 (see $\S$ III.B). In addition, models for kinematics solution of this mechanism are simple. However, the main weak point of I4 [10][11] is the use of prismatic joints in the articulated traveling plate. Indeed, used at high speed, commercial prismatic joints have a short service life, due to high acceleration and pressure exerted on balls. Thus, I4 is well suited for high force/ moderate acceleration application (machining for example).

For high speed robots, the use of revolute joints in the articulated traveling plate seems to be more adapted. That's why, Par4 has been developed with the constraint of using only revolute joints on its articulated traveling plate.

\section{B. From $\mathrm{H} 4$ to Par4}

H4 uses revolute joints, but a weak point of this robot is the arrangement of its actuators. This particularity is due to singularity configurations.
Singularities occur for particular poses of the mechanism and lead to a bad behavior of the end-effector. In [13], a classification of singularities is proposed. They can be parted into three categories: under-mobilities [14], over-mobilities [14], and internal singularities [15]. These notions can be partly explained using linear kinematic equation [16]:

$$
\boldsymbol{J}_{\boldsymbol{x}} \dot{\boldsymbol{x}}=\boldsymbol{J}_{q} \dot{\boldsymbol{q}}
$$

where $\dot{\boldsymbol{x}}$ is the vector of operational velocities and $\dot{\boldsymbol{q}}$ is the vector of actuated joints velocities.

In order to explain these categories of singularities, a simple two-dof parallel mechanism is proposed:

- On one hand, under-mobilities occur when $J_{q}$ is singular. In that case, a velocity $\dot{\boldsymbol{q}}$ can be applied without producing any motion on the traveling plate (Fig. 3a),

- On the other hand, over-mobilities occur when $\boldsymbol{J}_{\boldsymbol{x}}$ is singular. In that case, it is possible to have $\dot{\boldsymbol{x}} \neq \boldsymbol{0}$ without moving the actuators (Fig. 3b).

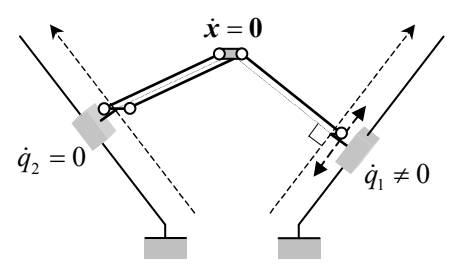

(a) Under-mobility configuration

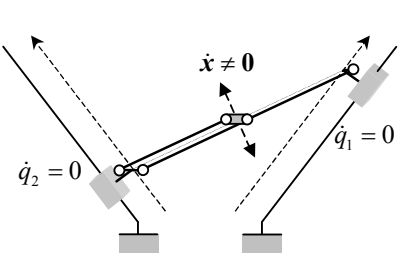

(b) Over-mobility configuration
Fig. 3 Presentation of singularities

At last, "internal" singularities can occur for some mechanisms. They cannot be enlightened thanks to $\boldsymbol{J}_{\boldsymbol{x}}$ or $\boldsymbol{J}_{\boldsymbol{q}}$, and a more complete study has to be done. On Fig. 4, an internal singularity occurs when the parallelogram becomes flat. In that case, orientation of traveling plate cannot be guaranteed (this orientation does not belong to operaltional velocities).

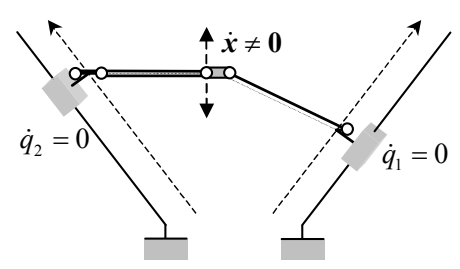

Fig. 4 Presentation of internal singularities

Placing actuators of $\mathrm{H} 4$ with a homogeneous repartition, i.e. placed at $90^{\circ}$ one relatively to each other, leads to internal singularities. Thus, a particular arrangement has to be adopted. This particularity involves a non-homogeneous behavior of $\mathrm{H} 4$ in the workspace and a bad stiffness, as demonstrated in [17].

As said before, the study of classical matrices $\boldsymbol{J}_{\boldsymbol{x}}$ and $\boldsymbol{J}_{\boldsymbol{q}}$ presented in equation (1) is not enough to enlighten internal singularities.

That's why, a complete kinematic analysis has been done on Par4 in order to show that this architecture overcomes 
drawbacks of $\mathrm{H} 4$ and $\mathrm{I} 4$ while keeping all the advantages for high speed. The method of the analysis has been introduced in [11] and [18] for I4 case. The analysis assumes that the mechanism is constituted of 2 sub-unit (actuators and traveling plate) linked by 8 rods having spherical joints. Each rod adds between those two sub-sets a pure geometrical length constraint.

This complete study can be done writing equiprojectivity of speeds for the 8 rods joining the actuators to the traveling plate. It leads to the following equation:

$$
J_{t p} \dot{x}_{1}=J_{a c t} \dot{q}
$$

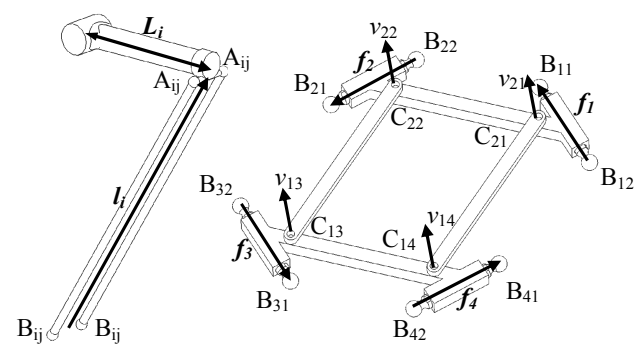

Fig. 5 Par4 parameters for complete singularity analysis

As shown in Fig. 5, the following parameters are introduced:

- $i$ : number of kinematic chain. $\mathrm{i}=1,4$

- $j$ : number of rod in each kinematic chain. $j=1,2$

- $k$ : number of half traveling plate. $\mathrm{k}=1,2$

- $\mathrm{A}_{i j}$ : center of spherical joints on actuated side of forearms

- $\mathrm{B}_{i j}$ : center of spherical joints on traveling plate side of forearms

- $\mathrm{A}_{i}$ : geometrical point situated at the middle of $\mathrm{A}_{\mathrm{i} 1}$ and $\mathrm{A}_{\mathrm{i} 2}$

- $\mathrm{B}_{i}$ : geometrical point situated at the middle of $\mathrm{B}_{\mathrm{i} 1}$ and $\mathrm{B}_{\mathrm{i} 2}$

$-\mathrm{C}_{i}$ : center of revolute joints of traveling plate

- D: controlled point (located on one of the parts of traveling plate)

- $\boldsymbol{l}_{i}$ : vector between $\mathrm{B}_{\mathrm{i}}$ and $\mathrm{A}_{\mathrm{i}}$

- $f_{i}$ : vector between $\mathrm{B}_{\mathrm{i} 1}$ and $\mathrm{B}_{\mathrm{i} 2}$

- $v_{k i}$ : unitary vector of collinear revolute joint axis $k i$

- $\boldsymbol{d}_{\mathrm{i}}$ : vector linking $\mathrm{C}_{\mathrm{i}}$ and $\mathrm{B}_{\mathrm{i}}$

- $c_{\boldsymbol{k}}$ : vector linking $\mathrm{C}_{\mathrm{i}}$ and $\mathrm{D}$

- $\boldsymbol{e}_{\boldsymbol{i}}=\boldsymbol{c}_{\boldsymbol{k}}+\boldsymbol{d}_{\mathrm{i}}$

- $\dot{\varepsilon}_{k i}$ :velocity of part $\mathrm{k}$ respectively to parallelogram rod (in revolute joint \# $k i$ oriented by $\boldsymbol{v}_{\boldsymbol{k}}$ )

- $\omega_{x}, \omega_{y}, \omega_{z}$ : internal angular velocities

- $\left(\boldsymbol{e}_{x}, \boldsymbol{e}_{y}, \boldsymbol{e}_{z}\right):$ reference frame axes, with $\boldsymbol{e}_{z}$ describing the vertical axis

Assuming that the PI joint in the traveling plate induces a constant parallelism of rods, it produces a coupling. Thus, velocities of revolute joints of traveling plate are equal and the following simplifications can be made:

$$
\begin{aligned}
& \dot{\varepsilon}_{14}=\dot{\varepsilon}_{13}=\dot{\varepsilon} \\
& \dot{\varepsilon}_{21}=\dot{\varepsilon}_{22}=\dot{\varepsilon}
\end{aligned}
$$

This coupling is the key difference with the complete kinematic modeling of $\mathrm{H} 4$.
Additionally, the following assumptions are made:

$$
\begin{aligned}
& v_{14}=v_{13}=e_{z} \\
& v_{21}=v_{22}=e_{z}
\end{aligned}
$$

Note that $\dot{\boldsymbol{x}}$ presented in (1) is the vector composed of operational velocities whereas $\dot{\boldsymbol{x}}_{\boldsymbol{l}}$ in (2) is the vector composed of velocities of the complete articulated traveling plate, including internal velocities. Thus, $\dot{\boldsymbol{x}}_{\boldsymbol{l}}$ is the following vector:

$$
\dot{\boldsymbol{x}}_{1}=\left[\begin{array}{lllllll}
\dot{x} & \dot{y} & \dot{z} & \omega_{x} & \omega_{y} & \omega_{z} & \dot{\varepsilon}
\end{array}\right]^{\mathrm{T}}
$$

where $\dot{x}, \dot{y}, \dot{z}, \omega_{z}$ are operational velocities.

It is now possible to write the $[8 \times 7]$ matrix $\boldsymbol{J}_{t p}$ as described in (8).

$$
\boldsymbol{J}_{\boldsymbol{t} p} \dot{\boldsymbol{x}}_{\boldsymbol{l}}=\left[\begin{array}{ccc}
\boldsymbol{l}_{1}^{T} & {\left[\boldsymbol{e}_{1} \times \boldsymbol{l}_{1}\right]^{T}} & \left(\boldsymbol{e}_{z} \times \boldsymbol{d}_{1}\right) \cdot \boldsymbol{l}_{1} \\
\boldsymbol{l}_{2}^{T} & {\left[\boldsymbol{e}_{2} \times \boldsymbol{l}_{2}\right]^{T}} & \left(\boldsymbol{e}_{z} \times \boldsymbol{d}_{2}\right) \cdot \boldsymbol{l}_{2} \\
\boldsymbol{l}_{3}^{T} & {\left[\boldsymbol{e}_{3} \times \boldsymbol{l}_{3}\right]^{T}} & \left(\boldsymbol{e}_{z} \times \boldsymbol{d}_{3}\right) \cdot \boldsymbol{l}_{3} \\
\boldsymbol{l}_{4}^{T} & {\left[\boldsymbol{e}_{4} \times \boldsymbol{l}_{4}\right]^{T}} & \left(\boldsymbol{e}_{z} \times \boldsymbol{d}_{4}\right) \cdot \boldsymbol{l}_{4} \\
\mathbf{0} & {\left[\boldsymbol{f}_{1} \times \boldsymbol{l}_{1}\right]^{T}} & \left(\boldsymbol{e}_{z} \times \boldsymbol{f}_{1}\right) \cdot \boldsymbol{l}_{1} \\
\mathbf{0} & {\left[\boldsymbol{f}_{2} \times \boldsymbol{l}_{2}\right]^{T}} & \left(\boldsymbol{e}_{z} \times \boldsymbol{f}_{2}\right) \cdot \boldsymbol{l}_{2} \\
\mathbf{0} & {\left[\boldsymbol{f}_{3} \times \boldsymbol{l}_{3}\right]^{T}} & \left(\boldsymbol{e}_{z} \times \boldsymbol{f}_{3}\right) \cdot \boldsymbol{l}_{3} \\
\mathbf{0} & {\left[\boldsymbol{f}_{4} \times \boldsymbol{l}_{4}\right]^{T}} & \left(\boldsymbol{e}_{z} \times \boldsymbol{f}_{4}\right) \cdot \boldsymbol{l}_{4}
\end{array}\right]\left[\begin{array}{c}
\dot{x} \\
\dot{y} \\
\dot{z} \\
\dot{\omega}_{x} \\
\dot{\omega}_{y} \\
\dot{\omega}_{z} \\
\dot{\varepsilon}
\end{array}\right]
$$

where $\times$ and $\cdot$ are respectively cross-product and dotproduct.

Matrix $\boldsymbol{J}_{\boldsymbol{t}}$ expressed in (8) can be manipulated in order to enlighten blocks. It is then possible de rewrite expression (2). It leads to the following equation:

$$
\left[\begin{array}{ccc}
\boldsymbol{l}_{1}^{T} & \left(\boldsymbol{c}_{1} \times \boldsymbol{l}_{1}\right) \cdot \boldsymbol{e}_{z} & {\left[\boldsymbol{e}_{1} \times \boldsymbol{l}_{1}\right]^{T}} \\
\boldsymbol{l}_{2}{ }^{T} & \left(\boldsymbol{c}_{1} \times \boldsymbol{l}_{2}\right) \cdot \boldsymbol{e}_{z} & {\left[\boldsymbol{e}_{2} \times \boldsymbol{l}_{2}\right]^{T}} \\
\boldsymbol{l}_{3} & \left(\boldsymbol{c}_{2} \times \boldsymbol{l}_{3}\right) \cdot \boldsymbol{e}_{z} & {\left[\boldsymbol{e}_{3} \times \boldsymbol{l}_{3}\right]^{T}} \\
\boldsymbol{l}_{4}{ }^{T} & \left(\boldsymbol{c}_{2} \times \boldsymbol{l}_{4}\right) \cdot \boldsymbol{e}_{z} & {\left[\boldsymbol{e}_{4} \times \boldsymbol{l}_{4}\right]^{T}} \\
\mathbf{0} & 0 & {\left[\boldsymbol{f}_{1} \times \boldsymbol{l}_{1}\right]^{T}} \\
\mathbf{0} & 0 & {\left[\boldsymbol{f}_{2} \times \boldsymbol{l}_{2}\right]^{T}} \\
\mathbf{0} & 0 & {\left[\boldsymbol{f}_{3} \times \boldsymbol{l}_{3}\right]^{T}} \\
\mathbf{0} & 0 & {\left[\boldsymbol{f}_{4} \times \boldsymbol{l}_{4}\right]^{T}}
\end{array}\right]\left[\begin{array}{c}
\dot{\boldsymbol{x}} \\
\omega_{z} \\
\omega_{x} \\
\omega_{y} \\
\omega_{z}+\dot{\varepsilon}_{1}
\end{array}\right]=\boldsymbol{J}_{q} \dot{\boldsymbol{q}}
$$

We recognize equation (1) with additional terms:

$$
\left[\begin{array}{cc}
J_{x} & J_{x}{ }^{i n t} \\
0 & J_{i n t}
\end{array}\right]\left[\begin{array}{c}
\dot{x} \\
v_{i n t}
\end{array}\right]=\left[\begin{array}{c}
J_{q} \dot{q} \\
0
\end{array}\right]
$$

With this modeling, $\boldsymbol{J}_{\text {int }}$ has the following expression:

$$
\boldsymbol{J}_{\boldsymbol{i n t}}=\left[\begin{array}{l}
{\left[\boldsymbol{f}_{1} \times \boldsymbol{l}_{1}\right]^{T}} \\
{\left[\boldsymbol{f}_{2} \times \boldsymbol{l}_{2}\right]^{T}} \\
{\left[\boldsymbol{f}_{3} \times \boldsymbol{l}_{3}\right]^{T}} \\
{\left[\boldsymbol{f}_{4} \times \boldsymbol{l}_{4}\right]^{T}}
\end{array}\right]
$$


and,

$$
\boldsymbol{v}_{\text {int }}=\left[\begin{array}{lll}
\omega_{x} & \omega_{y} & \left(\omega_{z}+\dot{\varepsilon}\right)
\end{array}\right]^{T}
$$

We can notice that the $[4 \times 3]$ matrix (11) shows that the mechanism is over-constrained (over-determined system). In addition, developing equation (10) leads to:

$$
\left\{\begin{aligned}
J_{x} \dot{x}+J_{x}^{i n t} v_{i n t} & =J_{q} \dot{q} \\
J_{i n t} v_{\text {int }} & =0
\end{aligned}\right.
$$

The second equation of system (13) shows that additional terms $\boldsymbol{v}_{\boldsymbol{i n t}}$ will be null if $\boldsymbol{J}_{\boldsymbol{i n t}}$ is a full-rank matrix. It means that mechanism will not have "internal singularities":

$$
\operatorname{rank}\left(\boldsymbol{J}_{\text {int }}\right)=3
$$

Additionally, the usual kinematic relation (1) will be derived.

Considering that the four pairs of rods have a symmetric contribution on the mechanism, (14) leads to calculate one determinant $D_{i j k}$ (among the four possible ones), and to verify that:

$$
\exists(i, j, k) \in\{(1,2,3),(1,2,4),(1,3,4),(2,3,4)\}, D_{i j k} \neq 0
$$

where,

$$
D_{i j k}=\left(\left(\boldsymbol{f}_{i} \times \boldsymbol{l}_{i}\right) \times\left(\boldsymbol{f}_{j} \times \boldsymbol{l}_{j}\right)\right) \cdot\left(\boldsymbol{f}_{k} \times \boldsymbol{l}_{k}\right)
$$

This working condition remains always true while placing the motors at $90^{\circ}$ one relatively to each other and scanning the whole workspace. This fact is a key issue for robot performance.

To summarize, Par4 has been developed with the aim of reaching high speed and acceleration. Thus, the adopted configuration is a good compromise between $\mathrm{H} 4$ and I4 robots: passive joints used in the articulated traveling plate are only revolute joints and motors are placed in homogenous arrangement.

\section{DyNAmic Modeling}

\section{A. Parameters and simplifications}

The following geometrical and dynamical parameters are introduced:
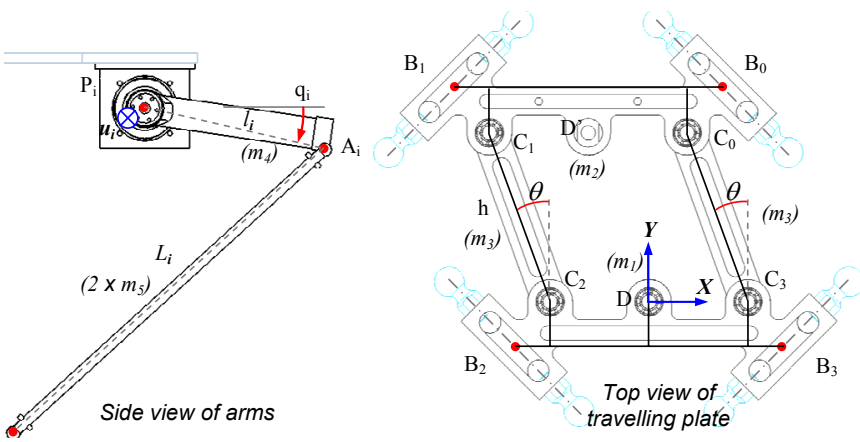

Fig. 6 Parameters used in dynamic modeling

- $\theta$ is the controlled angle in absolute coordinates,

- $\mathrm{h}$ is the length of the parallelogram of travelling-plate,

- $\boldsymbol{u}_{\boldsymbol{i}}$ is the unit vector collinear to actuator axis,
- $i_{a}$ : inertia of arms, $i_{f a}$ : inertia of forearms, $i_{m}$ : inertia of gears and actuator,

- $m_{1}$ : masse of first half traveling plate $\left(\mathrm{B}_{2} \mathrm{~B}_{3}\right.$ side $), m_{2}$ : masse of second half traveling plate $\left(\mathrm{B}_{0} \mathrm{~B}_{1}\right.$ side $), m_{3}$ : masse of rods of planar parallelogram of traveling plate, $m_{4}$ : masse of arms, $m_{5}$ : masse of forearms (of each rod)

- $\boldsymbol{I}_{a c t}$ : inertia matrix applied on actuators

- $\boldsymbol{M}_{\boldsymbol{1}}$ : masse matrix of first half traveling plate $\left(\mathrm{B}_{0} \mathrm{~B}_{1}\right.$ side $)$

- $\boldsymbol{M}_{2}$ : masse matri $\mathrm{x}$ of second half traveling plate $\left(\mathrm{B}_{2} \mathrm{~B}_{3}\right.$ side)

- $\boldsymbol{M}_{4}$ : masse matrix of arms

- $M_{5}$ : masse matrix of forearms

In order to compute the dynamic modeling, some simplifications have to be done. They are listed below:

i) the weight of spherical parallelograms are represented as two 'pinpoint' masses at each extremities,

ii) the inertia of rods of planar parallelogram of traveling plate is neglected,

iii) the weight of these rods represented as two 'pinpoint' masses at each extremities,

iv) the weight of these rods represented as two 'pinpoint' masses at each extremities

\section{B. Modeling}

This modeling permits to calculate torques applied to each actuator due to several contributions

\section{1) Torque due to inertias}

The torque due to inertias of actuators, gears, arms and forearms is defined by:

$$
\boldsymbol{\tau}_{1}=\boldsymbol{I}_{a c t} \ddot{\boldsymbol{Q}}
$$

with $I_{a c t}=\operatorname{diag}\left(\left[i_{a}+i_{f a}+i_{m}\right]\right)$ and $i_{f a}=m_{5} L^{2}$

2) Torque due to first half traveling plate

The contribution due to efforts applied on this half traveling plate is calculated using the jacobian matrix. This jacobian matrix is calculated on point $\mathrm{D}$, and is defined by the following expressions:

$$
\boldsymbol{J}_{1}=\boldsymbol{J}_{x 1}^{-1} \cdot \boldsymbol{J}_{\boldsymbol{q} 1}
$$

with

$$
\begin{aligned}
& \boldsymbol{J}_{\boldsymbol{x} 1}=\left[\begin{array}{cccc}
\boldsymbol{A}_{\theta} \boldsymbol{B}_{0} \cdot \boldsymbol{x} & \boldsymbol{A}_{\theta} \boldsymbol{B}_{0} \cdot \boldsymbol{y} & \boldsymbol{A}_{\theta} \boldsymbol{B}_{\theta} \cdot \boldsymbol{z} & -h \cos \theta \cdot \boldsymbol{A}_{\theta} \boldsymbol{B}_{\theta} \cdot \boldsymbol{x}-h \sin \theta \cdot \boldsymbol{A}_{\theta} \boldsymbol{B}_{0} \cdot \boldsymbol{y} \\
\boldsymbol{A}_{1} \boldsymbol{B}_{1} \cdot \boldsymbol{x} & \boldsymbol{A}_{1} \boldsymbol{B}_{1} \cdot \boldsymbol{y} & \boldsymbol{A}_{1} \boldsymbol{B}_{1} \cdot \boldsymbol{z} & -h \cos \theta \cdot \boldsymbol{A}_{1} \boldsymbol{B}_{1} \cdot \boldsymbol{x}-h \sin \theta \cdot \boldsymbol{A}_{1} \boldsymbol{B}_{1} \cdot \boldsymbol{y} \\
\boldsymbol{A}_{2} \boldsymbol{B}_{2} \cdot \boldsymbol{x} & \boldsymbol{A}_{2} \boldsymbol{B}_{2} \cdot \boldsymbol{y} & \boldsymbol{A}_{2} \boldsymbol{B}_{2} \cdot \boldsymbol{z} & 0 \\
\boldsymbol{A}_{3} \boldsymbol{B}_{3} \cdot \boldsymbol{x} & \boldsymbol{A}_{3} \boldsymbol{B}_{3} \cdot \boldsymbol{y} & \boldsymbol{A}_{3} \boldsymbol{B}_{3} \cdot \boldsymbol{z} & 0
\end{array}\right] \\
& \text { And } \\
& \boldsymbol{J}_{q 1}=\operatorname{diag}\left(\left[\left(\boldsymbol{A}_{\boldsymbol{i}} \boldsymbol{B}_{\boldsymbol{i}} \times \boldsymbol{P}_{\boldsymbol{i}} \boldsymbol{A}_{\boldsymbol{i}}\right) \cdot \boldsymbol{v}_{\boldsymbol{i}}\right]\right)
\end{aligned}
$$

The efforts applied to the half traveling plate are calculated using the following equation:

$$
F_{1}=M_{1}\left(\ddot{X}_{1}+g\right)
$$


As the half traveling has only translational motions, the acceleration is calculated as follow:

$$
\ddot{X}_{1}=T_{1} \ddot{X}
$$

where $\ddot{X}$ is operational accelerations, and

$$
\boldsymbol{T}_{1}=\left[\begin{array}{llll}
1 & 0 & 0 & 0 \\
0 & 1 & 0 & 0 \\
0 & 0 & 1 & 0 \\
0 & 0 & 0 & 0
\end{array}\right]
$$

In addition,

$$
\boldsymbol{M}_{1}=\left[\begin{array}{cccc}
m_{1}+m_{3}+2 m_{5} & 0 & 0 & 0 \\
0 & m_{1}+m_{3}+2 m_{5} & 0 & 0 \\
0 & 0 & m_{1}+m_{3}+2 m_{5} & 0 \\
0 & 0 & 0 & I_{1}
\end{array}\right] \text { (24) }
$$

Finally, torque applied to the actuators due to the first half traveling plate is defined by:

$$
\tau_{2}=J_{1}^{T} M_{1}\left(\ddot{X}_{1}+g\right)
$$

3) Torque due to second half traveling plate

${ }^{2}$ Efforts exhorted to the second half traveling plate will produce torques on actuators. Indeed, a new jacobian matrix (calculated in D') has to be calculated.

It is defined by:

$$
\boldsymbol{J}_{2}=\boldsymbol{J}_{x 2}^{-1} \cdot \boldsymbol{J}_{q 2}
$$

with,

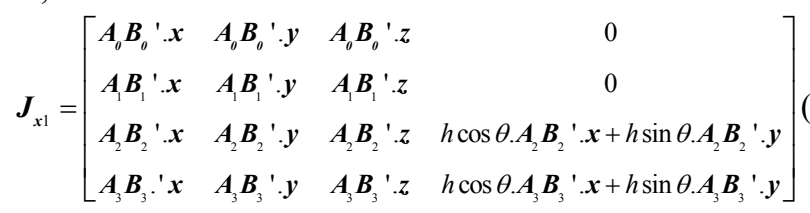

and,

$$
\boldsymbol{J}_{q 2}=\operatorname{diag}\left(\left[\left(\boldsymbol{A}_{\boldsymbol{i}} \boldsymbol{B}_{\boldsymbol{i}}{ }^{\prime} \times \boldsymbol{P}_{\boldsymbol{i}} \boldsymbol{A}_{i}^{\prime}\right) \cdot \boldsymbol{v}_{\boldsymbol{i}}\right]\right)
$$

In that case, the coordinates of vector $\boldsymbol{A}_{\boldsymbol{i}} \boldsymbol{B}_{\boldsymbol{i}}{ }^{\prime}$ and $\boldsymbol{P}_{\boldsymbol{i}} \boldsymbol{A}_{\boldsymbol{i}}{ }^{\prime}$ are calculated through absolute coordinates of $D^{\prime}$.

The efforts applied on this second half traveling plate are defined by:

$$
\boldsymbol{F}_{2}=\boldsymbol{M}_{2}\left(\ddot{\boldsymbol{X}}_{2}+\boldsymbol{g}\right)
$$

with,

$$
\boldsymbol{M}_{2}=\left[\begin{array}{cccc}
m_{2}+m_{3}+2 m_{5} & 0 & 0 & 0 \\
0 & m_{2}+m_{3}+2 m_{5} & 0 & 0 \\
0 & 0 & m_{2}+m_{3}+2 m_{5} & 0 \\
0 & 0 & 0 & I_{2}
\end{array}\right]
$$

and,

$$
\ddot{X}_{2}=T_{2} \ddot{X}+\dot{T}_{2} \dot{X}
$$

where,

$$
T_{2}=\left[\begin{array}{cccc}
1 & 0 & 0 & -h \cos \theta \\
0 & 1 & 0 & -h \sin \theta \\
0 & 0 & 1 & 0 \\
0 & 0 & 0 & 0
\end{array}\right]
$$

Finally, the torque due the second half traveling is calculated using this relation:

$$
\boldsymbol{\tau}_{3}=\boldsymbol{J}_{2}^{T} \boldsymbol{M}_{2}\left(\ddot{\boldsymbol{X}}_{1}+\boldsymbol{g}\right)
$$

4) Torque due to weights of arms and forearms

This contribution is calculated using the following relation:

$$
\tau_{4}=g L_{G} \sin \boldsymbol{Q} \boldsymbol{M}_{4}+g L \sin \boldsymbol{Q} \boldsymbol{M}_{5}
$$

Where $L_{G}$ is the distance between $P$ and the centre of mass of arms $\boldsymbol{P}_{\boldsymbol{i}} \boldsymbol{A}_{\boldsymbol{i}}$

5) Total torque

To conclude this dynamic modeling, the total torques applied to actuators is obtained by this relationship:

$$
\begin{gathered}
\boldsymbol{\tau}=\boldsymbol{I}_{\boldsymbol{a c t}} \ddot{\boldsymbol{Q}}+\boldsymbol{J}_{\boldsymbol{l}}{ }^{\boldsymbol{T}} \boldsymbol{M}_{\boldsymbol{1}}\left(\ddot{\boldsymbol{X}}_{\text {lin }}+\boldsymbol{g}\right)+\boldsymbol{J}_{2}{ }^{\boldsymbol{T}} \boldsymbol{M}_{2}\left(\ddot{\boldsymbol{X}}_{\text {lin } 2}+\boldsymbol{g}\right) \\
+g L_{G} \sin \boldsymbol{Q} \boldsymbol{M}_{4}+g L \sin \boldsymbol{Q} \boldsymbol{M}_{5}
\end{gathered}
$$

\section{Validation}

This modeling has been validated using Adams ${ }^{\circledR}$ software. The robot has been completely modeled, and a displacement has been controlled. The given torques has been compared to data given by the modeling described in this chapter.

Fig. 7 represents, in blue, torques obtained with Adams ${ }^{\circledR}$; and, in red, torques given by the modeling.
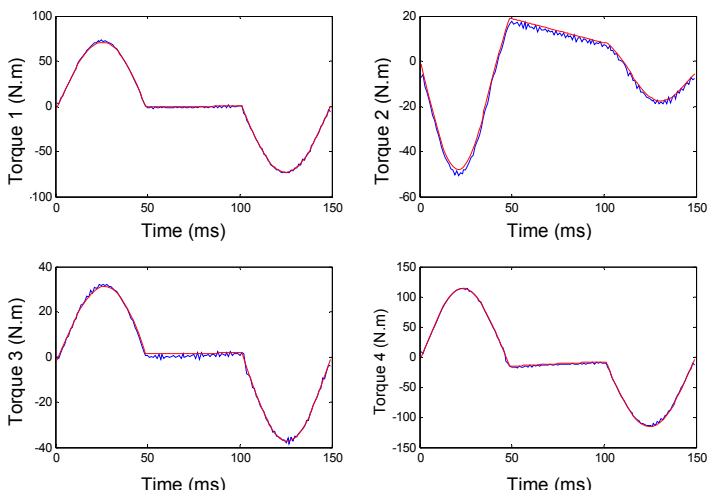

Fig. 7 Comparison of torques obtained by modelling and Adams ${ }^{\circledR}$

This comparison shows that the modelling is correct. In addition, it shows that simplifications done at $\S$ IV.A have very few consequences on final results.

\section{EXPERIMENTAL RESULTS}

All tests have been performed using Adept Motion (a classical industrial pick-and-place motion) with a length of $305 \mathrm{~mm}$ and an altitude of $25 \mathrm{~mm}$. The control loop is P/PI applied on actuated variables.

The first experimentations have been done with the following characteristics: maximal velocity $(\mathrm{v})=2.5 \mathrm{~m} \cdot \mathrm{s}^{-1}$, 
maximal acceleration (a) $=100 \mathrm{~m} \cdot \mathrm{s}^{-2}$. Obtained cycle time (round trip) is: $0.45 \mathrm{~s}$. Records of this experimentation are presented at Fig. 8.

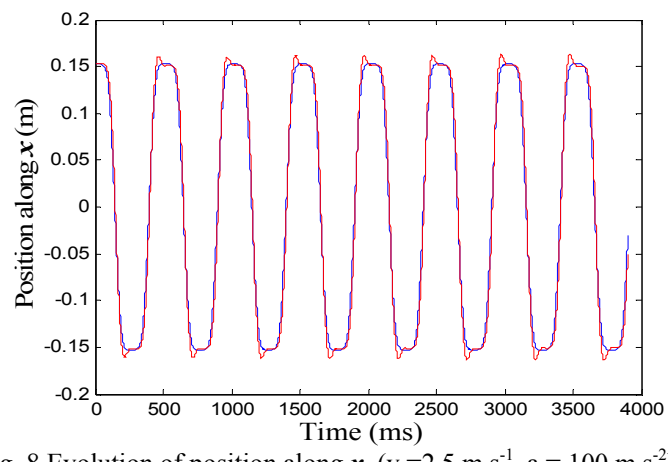

Fig. 8 Evolution of position along $x\left(\mathrm{v}=2.5 \mathrm{~m} \cdot \mathrm{s}^{-1}, \mathrm{a}=100 \mathrm{~m} \cdot \mathrm{s}^{-2}\right)$

The second experimentations have been made applying high speed and accelerations: $\mathrm{v}=3.8 \mathrm{~m} \cdot \mathrm{s}^{-1}, \mathrm{a}=130 \mathrm{~m} \cdot \mathrm{s}^{-2}$. The obtained cycle time is: 0.28 s. (see Fig. 9).

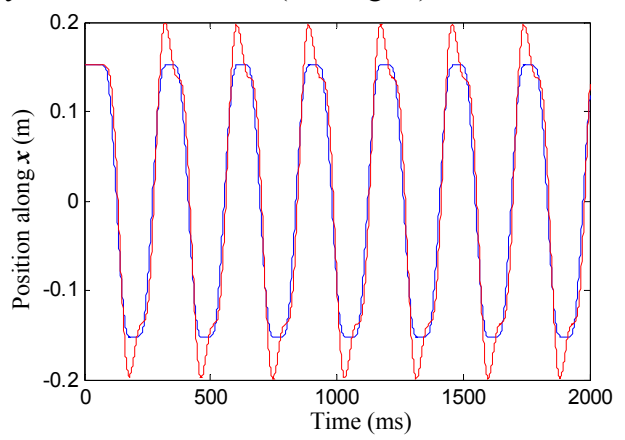

Fig. 9 Evolution of position along $x\left(v=3.8 \mathrm{~m} \cdot \mathrm{s}^{-1}, \mathrm{a}=130 \mathrm{~m} \cdot \mathrm{s}^{-2}\right)$

These experimentations show that we are able to have very good performances and to complete short cycle time. However, an overshoot is obtained as shown on Fig. 9. This restriction is partially due to torque limitation of actuators used on prototype.

\section{CONCLUSION}

This paper introduces a new four-dof parallel manipulator dedicated to pick-and-place and developed to perform high speed and acceleration. It shows that this robot is an improvement of H4, I4 and Delta robots and its architecture has been developed to overcome drawbacks of these existing robots. The key point of this architecture is its symmetrical arrangement of actuators (involving homogenous behavior) and the use of articulated traveling plate made of revolute joints. This key point has been proved making a complete analysis of all singularities. In addition, the dynamic modeling of the robot has been computed. This study will be useful for the future dynamic control that will be implemented on future works. The tests show that the prototype is able to reach an acceleration of $13 \mathrm{G}$ and a cycle time equal to $0.28 \mathrm{~s}$.

A demonstration video of Par4 prototype can be consulted at: http://www.lirmm.fr/ nabat/par4.wmv

\section{REFERENCES}

[1] V. E. Gough, "Contribution to discussion of papers on research in Automotive stability, control and tyre performance", in Proc. Auto. Div., Institute of mechanical engineering, 1956-1957.

[2] D. Stewart, "A platform with 6 degrees of freedom", in Proc. Inst. Mech. Ing., 1965, pp. 371-386, vol. 180, (part 1,15).

[3] T. Brogardh, "PKM Research - important issues, as seen from a product development perspective at ABB robotics", in Workshop on Fundamental Issues and Future Research Directions for Parallel Mechanisms and Manipulators. Quebec City, Quebec, Canada: Clément M. Gosselin and Imme Erbert-Uphoff, editors, 2002.

[4] J. M. Hervé, "The lie group of rigid body displacements, a fundamental tool for mechanism design”, Mechanism and Machine Theory, vol. 34, pp. 719-730, 1999.

[5] R. Clavel, "Delta, a fast robot with parallel geometry", in 18th Int.Symp. on Industrial Robots. Lausanne: IFS Publications, april 1988, pp. 91-100.

[6] J. Angeles, A. Morozov, and O. Navarro, "A novel manipulator architecture for the production of scara motions", in Proceedings IEEE International Conference on Robotics and Automation, San Francisco, USA, April 2000, pp. 2370-2375, CDROM.

[7] L. Rolland, "The manta and the kanuk: Novel 4-dof parallel mechanisms for industrial handling", in ASME Dynamic Systems and Control Division, IMECE'99 Conference, Nashville, USA, November 1999, pp. 831-844.

[8] M. Thurneysen, M. Schnyder, R. Clavel, and J. Jiovanola, "A new parallel kinematics for high speed machine tools hita stt", in 3rd Chemnitzer Parallelkinematik Seminar (PKS 2002), Chemnitz, Germany, april 23-25 2002 , pp. $553 \tilde{n} 562$.

[9] F. Pierrot and O. Company, "H4: a new family of 4-dof parallel Robots", in AIM'99: IEEE/ASME International Conference on Advanced Intelligent Mechatronics, Atlanta, Georgia, USA, September 1999, pp. 508-513.

[10]S. Krut, O. Company, M. Benoit, H. Ota, and F. Pierrot, "I4: A new parallel mechanism for SCARA motions", in Proc. of IEEE ICRA: Int. Conf. on Robotics and Automation, Taipei, Taiwan, September 14-19 2003.

[11]S. Krut, V. Nabat, O. Company, and F. Pierrot, "A high speed robot for scara motions", in Proc. of IEEE ICRA: Int. Conf. on Robotics and Automation, New Orleans, USA, April 26 - May 12004.

[12]J. M. Hervé and F. Sparacino, "Star, a new concept in robotics", in IMACS/SICE International Symposium on Robotics, Mecatronics and Manufacturing Systems, vol 1, Kobe, Japan, September 1992, pp. 176183.

[13]D. Zlatanov, R. G. Fenton, and B. Benhabib, "Identification and classification of the singular configurations of mechanisms", in Mechanism and Machine Theory, Vol. 33, No. 6, pp. 743-760, August 1998.

[14]C. Gosselin, and J. Angeles, "Singularity Analysis of Closed-Loop Kinematic Chains", in IEEE Transactions on Robotics ans Automation, Vol.6, n³, pp.281-290, June 1990.

[15]D. Zlatanov, I. Bonev, and C. Gosselin, "Constraint Singularities", Laboratoire de l'Université de Laval, Québec, Web review, 2001.

[16]C. Gosselin, "Kinematic analysis optimization and programming of parallel robotic manipulators", Ph.D Thesis, McGill University, Montreal, 1988.

[17]O. Company, F. Pierrot, and J. C. Fouroux, "A Method for Modeling Analytical Stiffness of a Lower Mability Parallel Manipulator', in Proc. Of IEEE ICRA: Int. Conf. on Robotics and Automation, Barcelona, Spain, 18-22 April 2005

[18]S. Krut, "Contribution à l'Etude des Robots Parallèles Légers, 3T-1R et 3T-2R, à Forts Débattements Angulaires", Ph.D Thesis, Université Montpellier II Sciences et Techniques du Languedoc, Montpellier, 2003.

[19]M. R. Spiegel, "Formules et tables mathématiques", Schaum, McGrawHill Inc., 1979 